\title{
Posterior implementation vs ex-post implementation is
}

\author{
Philippe Jehiel $^{\mathrm{a}, \mathrm{b}}$, Moritz Meyer-ter-Vehn ${ }^{\mathrm{c}}$, \\ Benny Moldovanu ${ }^{\mathrm{d}, *}$, William R. Zame ${ }^{\mathrm{e}}$ \\ ${ }^{\text {a }}$ UCL, United Kingdom \\ ${ }^{\mathrm{b}}$ PSE, France \\ ${ }^{\mathrm{C}}$ SAP, Germany \\ d Department of Economics, University of Bonn, Lennstr. 37, 53113 Bonn, Germany \\ ${ }^{\mathrm{e}}$ UCLA, United States
}

Received 1 September 2006; received in revised form 24 January 2007; accepted 14 February 2007

Available online 21 June 2007

\begin{abstract}
Posterior implementation is a weaker concept than ex-post implementation. It requires that agents' strategies are optimal against others' strategies, given the precise information made available by the mechanism. Whereas expost implementation is generically impossible, we show by example that this need not be the case for posterior implementation.
\end{abstract}

(C) 2007 Elsevier B.V. All rights reserved.

Keywords: Posterior implementation; Ex-post implementation; Multi-dimensional types

JEL classification: D44; D81

In this short note we discuss how posterior implementation, due to Green and Laffont (1987), relates to ex-post implementation for which Jehiel et al. (2006) have established a strong impossibility result.

\footnotetext{
Financial support provided by the Max Planck Research Prize (Moldovanu) and by the John Simon Guggenheim Foundation, the National Science Foundation (under grants SES-0079299 and SES-0317752) and the UCLA Academic Senate Committee on Research (Zame).

* Corresponding author. Tel.: +49 228 736395; fax: +49 228737940.

E-mail address: mold@uni-bonn.de (W.B. Moldovanu).
}

0165-1765/\$ - see front matter (C) 2007 Elsevier B.V. All rights reserved. doi:10.1016/j.econlet.2007.02.023 
In contrast to most other notions of implementation, posterior implementation is defined with respect to the information released by the mechanism. It requires that agents' strategies are optimal against others' strategies, given the precise information made available by the mechanism. It can be interpreted as a regretfree form of implementation: even after agents gain new information by observing some specified features of the mechanism's outcome (usually the equilibrium action profile), they are still willing to stick to their original strategy.

This notion of regret-free implementation (which can be viewed as robustness with respect to the timing of choice) is different from the notion of robust implementation that refers to the agent' prior beliefs and higher-order beliefs. As shown by Bergemann and Morris (2005), robust implementation implies ex-post implementation in a large class of environments with quasi-linear preferences, and thus it constitutes a main motivation for ex-post implementation.

Despite the stark difference in interpretation, note that, if the information released by the mechanism includes all players' types, then posterior implementation coincides with ex-post implementation. At the other extreme, if no further information is made available to agents, then posterior implementation coincides with Bayes-Nash implementation.

Particularly in settings where signal spaces are of higher dimension than actions spaces, some form of posterior implementation may be possible even if ex-post implementation fails (since in such settings agents are able to make only limited inferences about the signals behind particular actions). We illustrate this phenomenon below.

\section{Example 0.1.}

- There are two bidders $i \in\{1,2\}$;

- Bidders have two-dimensional signals $s^{i}=\left(p^{i}, c^{i}\right) \in[0,1]^{2}$;

- The valuation of bidder $i$ for the unit is given by $v^{i}\left(s^{i}, s^{-i}\right)=p_{i}+c^{i} c^{-i}$. Assume that the signals $\left(p^{i}, c^{i}\right)$ are uniformly and independently distributed on $[0,1]^{2}$.

Claim 0.2. The second-price auction has a symmetric Bayes-Nash equilibrium with an associated nontrivial allocation function.

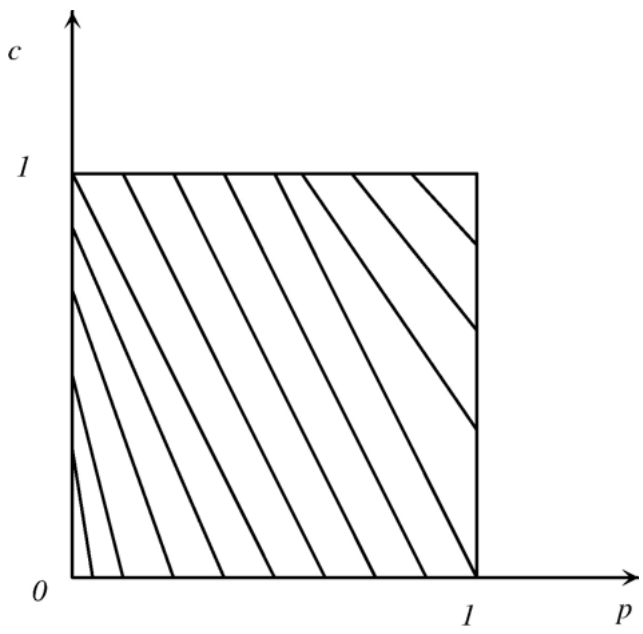

Fig. 1. Iso-bids in the second-price auction. 
Proof. We construct a symmetric equilibrium in continuous, strictly monotonic increasing bid functions $b:[0,1]^{2} \rightarrow \mathbb{R}$. A necessary and sufficient condition for equilibrium is that each type $(p, c)$ is indifferent between winning or losing the auction at a tie. This gives the usual condition:

$$
b=b\left(s^{i}\right)=\mathbb{E}_{s^{-i}}\left[v^{i}\left(s^{i}, s^{-i}\right) \mid b\left(s^{-i}\right)=b\left(s^{i}\right)\right]
$$

Given signals' independence, the right hand side is equal to $p^{i}+c^{i} \bar{c}(b)$. Here, $\bar{c}(x)=\mathbb{E}_{S^{-} i}[c \mid b(p, c)=x]$ is the expectation of the opponent's common values signal given that he makes bid $x$. This shows that the iso-bid curves $b^{-1}(x)$ must be straight lines with slope $-\frac{1}{\bar{c}(x)}$ in the $(p, c)$-space $[0,1]^{2}$. Some tedious calculations show that the iso-bid lines are as follows:

$$
b^{-1}(x):= \begin{cases}{[(0, \sqrt{2 x}),(x, 0)]} & \text { for } x \in\left[0, \frac{1}{2}\right] \\ {\left[\left(x-\frac{1}{2}, 1\right),(x, 0)\right]} & \text { for } \mathrm{x} \in\left[\frac{1}{2}, 1\right] \\ {\left[\left(x^{-} \frac{1}{4}-\frac{1}{4} \sqrt{8 x-7}, 1\right),\left(1, \frac{1}{2} \sqrt{8 x-7}-\frac{1}{2}\right)\right]} & \text { for } \mathrm{x} \in[1,2] .\end{cases}
$$

These iso-bid lines are drawn in Fig. 1.

Claim 0.3. The above exhibited non-trivial equilibrium is a posterior equilibrium assuming that bids (but nothing else) are made public after the auction. On the other hand, only trivial allocation functions are ex-post implementable. ${ }^{1}$

Proof. Posterior implementation with respect to this disclosure policy requires optimality of each player's bid given other players' actual (rather than expected) bids. It is readily verified that the equilibrium exhibited above has this property: conditional on observing the bid $x$ of bidder $-i$, bidder $i$ makes the inference that the expected value of $c^{-i}$ is $E_{s^{-} i}\left[c^{-i} \mid b\left(p^{-i}, c^{-i}\right)=x\right]$, and therefore she is not willing to modify her bid.

It is easily checked that the above is not an ex-post equilibrium: assume that $(-i)$ 's signal is $s^{-i}=\left(p^{-i}, c^{-i}\right)$, while $i$ 's signal $s^{i}=\left(p^{i}, c^{i}\right)$ satisfies $p^{i}+c^{i} c^{-i}-b\left(s^{-i}\right)>0$ and $b\left(s^{i}\right)<b\left(s^{-i}\right)$. Then, after being informed about $(-i)$ 's signal, $i$ prefers bidding above $b\left(s^{-i}\right)$ rather than $b\left(s^{i}\right)$. The general impossibility follows by an application of Proposition 3.3 of Jehiel et al. (2006): Define the two alternatives " $i$ gets the object" and " $(-i)$ gets the object". The relative valuations are given by $\mu^{i}=p^{i}+c^{i} c^{-i}$ and $\mu^{-i}=-p^{-i}-c^{i} c^{-i}$. Assume that $(\psi, t)$ is a non-trivial ex-post incentive compatible mechanism with continuous relative transfers . Condition (1) of that Proposition requires the existence of an indifference signal $\hat{s} \in(0,1)^{4}$, of a vector $\left(y_{a}, y_{b}\right)^{T}$, and of a function $\lambda\left(c^{-i}\right) \in \mathbb{R}^{+}$such that:

$$
\lambda\left(c^{-i}\right)\left(\begin{array}{c}
1 \\
c^{-i}
\end{array}\right)=\left(\begin{array}{c}
0-y_{a} \\
-c^{-i}-y_{b}
\end{array}\right)
$$

\footnotetext{
${ }^{1}$ If the object is allowed to stay with the seller (an alternative constantly valued at zero by both buyers), Bikhchandani (2006) observes that ex-post implementation is possible in this example.
} 
for all $c^{-i}$ in a neighborhood of $\hat{c}^{-i}$. A consideration of the cross product $c^{-i}-y_{b}+y_{a} c^{-i}=0$ yields $y_{b}=0$ and $y_{a}=1$. This shows that $\nabla_{s i} \mu^{i}(s)$ and $\left(\nabla_{s i} \mu^{-i}(s)-(1,0)^{T}\right)$ are co-linear (but point in opposite directions) $)^{2}$.

For any setting where Bayes-Nash implementation of some social choice function is possible with respect to some priors, but where ex-post implementation fails, there will be some "maximal information mechanism" that allows for posterior implementation. It will be interesting to study the properties of such mechanisms.

\section{References}

Bergemann, D., Morris, S., 2005. Robust mechanism design. Econometrica 73, 1771-1813.

Bikhchandani, S., 2006. Ex-post implementation in environments with private goods. Theoretical Economics 1, $369-393$.

Green, J., Laffont, J.J., 1987. Posterior implementation in a two-person decision problem. Econometrica 55, 69-94.

Jehiel, P., Meyer-ter-Vehn, M., Moldovanu, B., Zame, W., 2006. The limits of ex post implementation. Econometrica 74, 585-611.

\footnotetext{
${ }^{2}$ To see that condition (2) of that Proposition isn't satisfied either, note that the direction of $\nabla_{s i} \mu^{i}(s)=\left(1, c^{-i}\right)^{T}$ cannot be locally independent of $s^{-i}$. Thus, non-trivial implementation fails also with discontinuous transfers.
} 